\title{
Use of 18S-rRNA-targeted oligonucleotide probes for detection and quantification of anaerobic fungi in the rumen of different animals
}

\author{
L Millet, G Fonty, Ph Gouet \\ INRA, Laboratoire de Microbiologie, C.R. de Clermont-Ferrand-Theix, \\ 63122 Saint-Genès-Champanelle, France
}

\begin{abstract}
Strictly anaerobic fungi are natural inhabitants of both the foregut (i.e. the rumen) and the hindgut (i.e. caecum) of a wide range of herbivorous mammals. The role of these fungi in fibre degradation, their phylogeny and life cycles have been the subject of numerous studies (Fonty and Joblin, 1991, in: Physiological aspects of digestion and metabolism in ruminants, Tsuda et al, eds, 655-680). However, the estimation of their populations in the rumen still presents a significant problem. Because of the close physical association of the fungal rhizoïds with plant material and the irregular production of zoospores, zoospore population densities (estimated at $10^{3}-10^{4} \mathrm{ml}^{-1}$ rumen contents) can only give an approximate indication of the total fungal biomass present. No specific culture media for detecting and counting the various fungal species exist and no appropriate chemical marker for fungal biomass has been identified to date. Modern molecular techniques offer the promise of an accurate and reliable method for both determinative and quantitative evaluation of the fungal population in the gut. Species specific DNA probes have been used to enumerate bacteria in the rumen (Stahl et al, 1988, Appl Environ Microbiol, 54, 1079-1084) and this technology should be applicable to the anaerobic fungi. Thus, the aim of this work was to evaluate the use of specific 18S rRNAtargeted oligonucleotide probes to detect and to quantify several phylogenetically-defined groups of fungi in the rumen of domestic and wild animals.
\end{abstract}

The animals used included 2 cows, 2 sheep and 2 llamas fed alfalfa hay, 1 deer and 2 bisons fed a grass based diet. Rumen samples (25 to $30 \mathrm{~g}$ of contents) were collected through the rumen canula from cows, sheep and lamas and just after slaughter from deer and bisons, frozen at $-80^{\circ} \mathrm{C}$ and then freezedried. Four ${ }^{32} \mathrm{P}$-labeled oligonucleotide probes were used, one targeting the total fungal population (probe 715 ), one the genus Caecomyces, one the genus Anaeromyces and one targeting both the Orpinomyces and Neocallimastix genera. The design of these probes was previously given (Dore et al, in: Manipulation of rumen microorganisms, ElShazly, ed, 336-337). The techniques used for labeling these probes, RNA extraction and dotblot hybridization were those of Stahl et al (1988). After dot-blot hybridization, bound probes were quantified by liquid-scintillation counting. The proportion of each specific group is expressed as a percentage of the total fungal 18S-rRNA quantified with probe 715 using standard RNA extracted from pure fungal strains.

Except in one sheep harbouring essentially the genus Anaeromyces ( $86 \%$ ), in all animals the fungal population was mainly composed by the genera Caecomyces (40-80\%) and Anaeromyces (30-55\%). Caecomyces was particularly abundant in bisons (70-80\%). In contrast the genera Orpinomyces and Neocallimastix were only detected as traces $(<5 \%)$. These results contrast with previous data obtained by culture techniques for enumerating zoospores in rumen fluid (Grenet et al, 1989, Appl Environ Microbiol, 55, 2360 2364; Breton et al, 1994, Ann Zootech, 43, 262) which indicated that Neocallimastix was more abundant than Caecomyces in the rumen. 